\title{
Phase-matched second-harmonic generation in bulk azodye-doped polymers by all-optical poling
}

\section{$\operatorname{AUTHOR}(S)$ :}

Si, Jinhai; Qiu, Jianrong; Zhai, Jianfeng; Shen, Yuquan; Meng, Zhichong; Hirao, Kazuyuki

\section{CITATION:}

Si, Jinhai ... [et al]. Phase-matched second-harmonic generation in bulk azodye-doped polymers by all-optical poling. JOURNAL OF APPLIED PHYSICS 2004, 95(7): 3837-3839

\section{ISSUE DATE:}

2004-04-01

URL:

http://hdl.handle.net/2433/39709

\section{RIGHT:}

Copyright 2004 American Institute of Physics. This article may be downloaded for personal use only. Any other use requires prior permission of the author and the American Institute of Physics. 


\title{
Phase-matched second-harmonic generation in bulk azodye-doped polymers by all-optical poling
}

\author{
Jinhai Si and Jianrong Qiu \\ Photon Craft Project, ICORP, JST, Keihanna-plaza, Super-lab 2-5, 1-7 Hikaridai, Seika-cho, \\ Kyoto, 619-0237, Japan \\ Jianfeng Zhai and Yuquan Shen \\ Technical Institute of Physics and Chemistry, Chinese Academy of Sciences, Beijing 100101, China \\ Zhichong Meng and Kazuyuki Hirao \\ Division of Material Chemistry, Faculty of Engineering, Kyoto University, Sakyo-ku Kyoto 606-8501, Japan
}

(Received 4 August 2003; accepted 19 January 2004)

\begin{abstract}
We demonstrate phase-matched second-harmonic generation (SHG) in bulk azodye-doped polymethylmethacrylate using an all-optical poling technique. During the seeding process, samples were irradiated simultaneously by coherent superposition of the fundamental and the second-harmonic light of a nanosecond laser. The measurements for the dependence of SHG on the sample thickness show that the SHG signals increase with the increase in thickness of the samples, indicating that a $\chi^{(2)}$ grating that satisfies the phase-matching condition for SHG could be optically induced in the polymer samples. (C) 2004 American Institute of Physics.
\end{abstract}

[DOI: $10.1063 / 1.1667271]$

Second-order nonlinear optical (NLO) polymers are very attractive for applications in optical communications and in high-density optical data storage. ${ }^{1,2}$ Poling techniques are usually used to realize the second-order NLO function of the polymer films, and all-optical poling has been demonstrated in azoaromatic acrylic copolymers using a dual frequency laser. $^{3-5}$ The physical mechanism of the effect consists of two processes: orientational hole burning and reorientation of azodye molecules. ${ }^{3}$ Under the excitation of a fundamental light together with its second-harmonic light, orientational hole burning of azodye molecules occurs through the interference of two-photon absorption at the fundamental frequency and one-photon absorption at the doubling frequency. Orientational hole burning is followed by reverse trans-cistrans isomerization, which finally leads to a net permanent polar orientation of molecules. This technique possesses some of the following advantages: phase matching for second-harmonic generation (SHG) can automatically be achieved, no electrodes are required, and micropatterning of the second-order susceptibility can be simply achieved by scanning the focal area over the sample surface.

A medium of millimeter thickness or more that exhibits a high second-order NLO coefficient is required to achieve high SHG conversion efficiency. However, in most up-todate studies on all-optical poling, the thickness is of the order of micrometers, due to the intense absorption of disperse red 1 (DR1) used as the second-order NLO materials for the 532-nm seed light of a Nd:YAG laser. ${ }^{6-9}$ Fiorini et al. presented nonresonant all-optical poling based on multiphoton excitation to overcome the absorption of samples for the 532-nm seed light, and obtained an photoinduced $\chi^{(2)}$ of $10^{-3} \mathrm{pm} / \mathrm{V}$ using a Nd:YAG laser delivering 30-ps pulses. ${ }^{10}$ The small photoinduced $\chi^{(2)}$ was probably due to the lower photoinduced molecular orientation $(\sim 0.1 \%)$ under the multiphoton excitation. To increase the photoinduced molecular orientation efficiency, we achieved nonresonant all-optical poling of thick azodye-doped polymethylmethacrylate (PMMA) films using the $1500-\mathrm{nm}$ fundamental and $750-\mathrm{nm}$ second-harmonic light of a femtosecond laser with high peak power, and obtained an SHG efficiency as high as $2 \%$. $^{11}$ Because of the group velocity mismatch between seed beam $\omega$ and seed beam $2 \omega$, however, the optimum thickness of samples was only $105 \mu \mathrm{m}$, and samples thicker than that caused the photoinduced molecular orientation efficiency to decrease.

We have prepared bulk azodye-doped PMMA samples, whose absorption is weak at the wavelength of the 532-nm seed light. This weak absorption should permit the use of bulk polymer samples in all-optical poling. In this paper, we report on phase-matched SHG in bulk azodye-doped PMMA by all-optical poling. During the seeding process, samples were irradiated simultaneously by coherent superposition of the fundamental and the second-harmonic light of a nanosecond Nd:YAG laser. The measurements for the dependence of SHG on the sample thickness showed that the SHG signals increased with the increase of thickness of the samples.

The azodye used in this work was 4-[4-(N-n-hexyl-Nmethylamino)-phenylazo]-benzoic acid (HPNA), which was synthesized by a general diazonium coupling reaction of $\mathrm{N}$-n-hexyl-N-methylaminobenzene and 4-diazonium salt of 4-aminobenzoic acid. The molecular structure of the HPNA azodye is shown in Fig. 1(a). Details of the preparation of the azodye-doped PMMA bulk samples were described in the literature. ${ }^{12}$ The weight ratio of the azodye and PMMA was $1.2 \%$; the molecular weights of the PMMA polymer were in the range of $60000-80000$. The size of the samples was about $10 \times 18 \mathrm{~mm}$, and the sample thickness ranged from 0.1 to $1 \mathrm{~mm}$. No substrate was used in the bulk samples. The samples had fairly good surface flatness. The absorption spectrum of a 1-mm-thick sample is shown in Fig. 1(b), from 


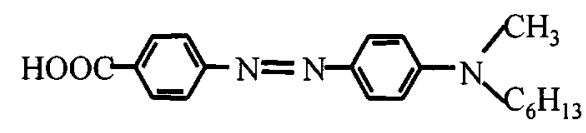

(a)

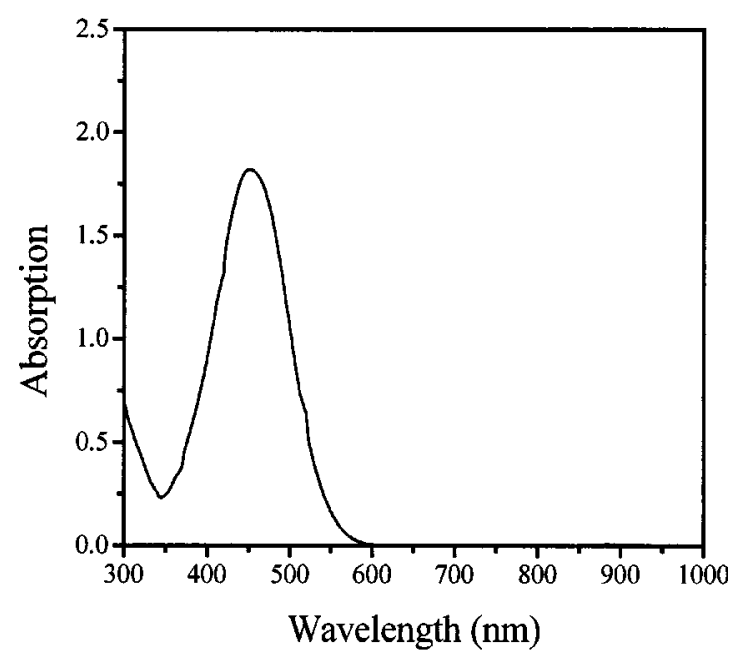

(b)

FIG. 1. The molecular structure (a) and absorption spectrum (b) of the HPNA azodye.

which one can see that the absorption band peaks at $450 \mathrm{~nm}$, and that the absorption coefficient of the sample was 5.9 $\mathrm{cm}^{-1}$ at a wavelength of $800 \mathrm{~nm}$.

A similar experimental setup as that in Ref. 13 was used. The light source was a Q-switched Nd:YAG laser, which emitted 3.5-ns, 1064-nm and 3-ns, 532-nm laser pulses, with a repetition rate that could be varied from 1 to $100 \mathrm{~Hz}$. The $\omega$ and $2 \omega$ light beams (which we refer to as the seed beams) were used for the preparation. During the preparation process, the two collinear seed beams were simultaneously incident upon the sample. During the probe, the $2 \omega$ beam was blocked by a shutter with only the $\omega$ beam remaining incident. The SHG signals of the $\omega$ beam were detected by a photomultiplier and observed and averaged by an oscilloscope. The $\omega$ beam passing through the sample was blocked by a heat-absorbing filter placed behind the sample, allowing only the SHG signal to pass through it. Typically, the pulse energies were about $1 \mathrm{~mJ}$ for the infrared $\omega$ beam and $1 \mu \mathrm{J}$ for the green $2 \omega$ beam, and the beam waists ( $1 / \mathrm{e}^{2}$ of intensity radius) at the sample were 400 and $350 \mu \mathrm{m}$, respectively. The poled area was approximately $0.5 \mathrm{~mm}^{2}$. In all optical poling processes, the seeding was performed at room temperature.

Figure 2 shows the growth and decay processes of photoinduced $\chi^{(2)}$ of a 1-mm-thick sample. Results show that when switching on the two seed beams the photoinduced $\chi^{(2)}$ reached its saturation value in 9 min for the condition described above. After the $\omega$ seed beam was switched off, the photoinduced $\chi^{(2)}$ decreased to $70 \%$ of its saturation value in about $20 \mathrm{~min}$. The decay shown in Fig. 2 was partly due to the photostimulated relaxation by the $\omega$ light. After the poled sample had been kept in the dark for $12 \mathrm{~h}$ at room temperature, the photoinduced $\chi^{(2)}$ decayed to $58 \%$ of its saturation

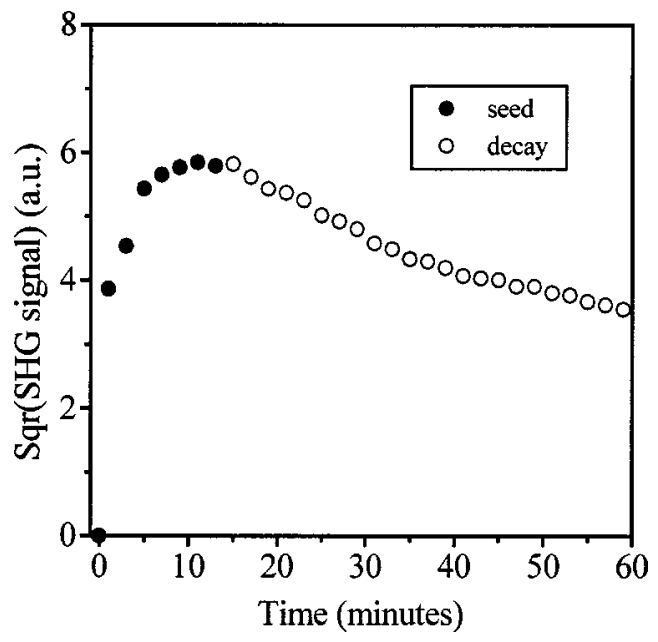

FIG. 2. Growth and decay of the photoinduced SHG signals of the 1-mmthick sample.

value. Our previous results have shown that the photoinduced orientation stability of azodye molecules in polymers could be improved by choosing thermosetting materials and employing the thermal-assisted photoinduced effects. ${ }^{13}$ In the thermal-assisted photoinduced process, the samples heated in an oven are irradiated with the writing beam; thus, the rotational relaxation of the azodye molecules can be restricted by the thermosetting matrix.

We also measured the dependence of the SHG signal intensity versus the sample thickness. The results are shown in Fig. 3, where all of the measurements were made after the photoinduced $\chi^{(2)}$ of the films increased to their saturation values. Figure 3 shows that the SHG signals increased with the increase in thickness of the samples. To justify that the SHG signal resulted from the phase-matched contribution, a theoretical fit based on phase-matched SHG from a medium with absorption was performed, where we used the following equation: ${ }^{5}$

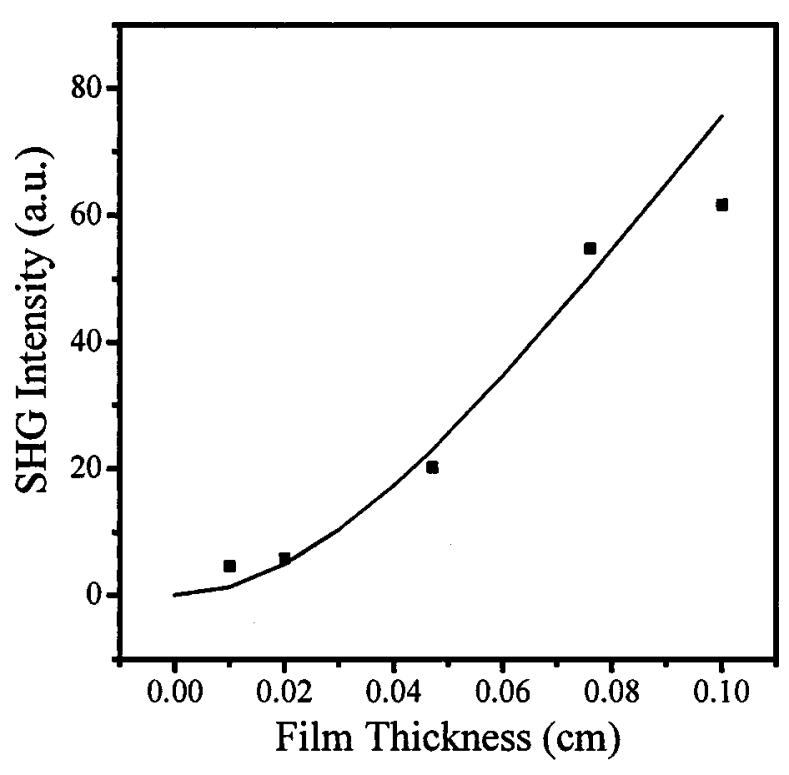

FIG. 3. The sample thickness dependence of the SHG intensity. The solid curve is a theoretical fit based on phase-matched SHG from a medium with absorption for the experimental data. 


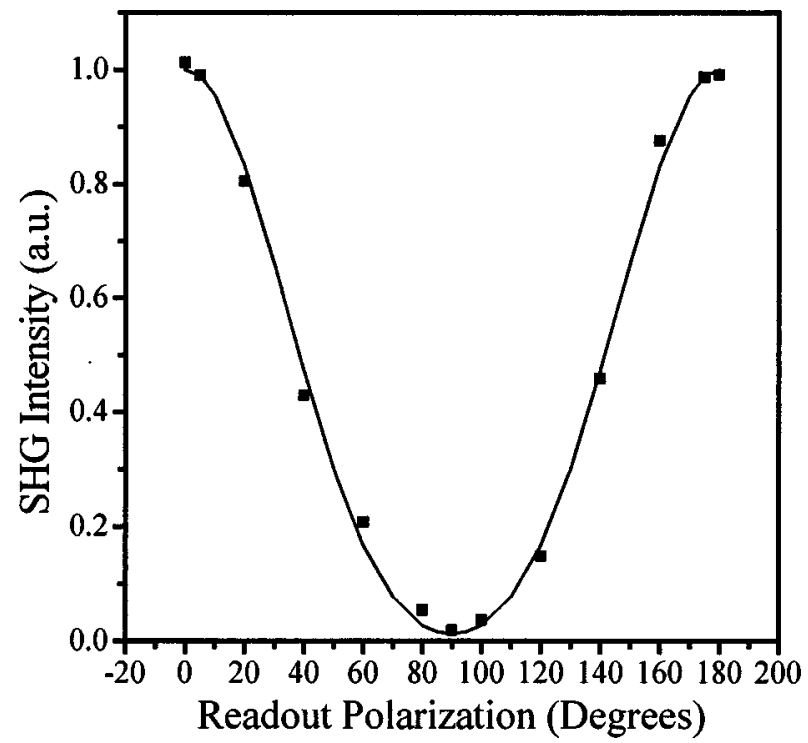

FIG. 4. Tensor data and fits for the $x$ component of SHG signals from the photoinduced $\chi^{(2)}$ of the 1-mm-thick sample.

$$
I_{2 \omega}=\frac{\omega^{2} d_{\mathrm{eff}}^{2}}{4 n^{2} c^{2} 10^{\mathrm{OD}}} I_{\omega}^{2} l^{2}
$$

where $I_{2 \omega}$ and $I_{\omega}$ are the SHG signal intensity and the input intensity of the $\omega$ beam, respectively. Here, $l$ is the sample thickness, and $d_{\text {eff }}$ is the effective second-order nonlinear coefficient of the sample; OD and $n$ are the optical absorption density and the refractive index of the sample at frequency $2 \omega$, respectively, and $c$ is the velocity of light. The results of the theoretical fit are also shown in Fig. 3, where the parameters in Eq. (1) were chosen as follows: $d_{\text {eff }}$ $=0.2 \mathrm{pm} / \mathrm{V}, n=1.5, \mathrm{OD}=0.25, I_{\omega}=0.3 \mathrm{GW} / \mathrm{cm}^{2}$. Experimental data are in good agreement with the theoretical estimation; in fact, phase-matched SHG for all-optical poling is a photoinduced quasi-phase-matched SHG. Under the excitation of a fundamental light along with its second-harmonic light, a quasi-phase-matched structure with the inverted domain grating, due to polar orientation modulation of azodye molecules, was induced in the polymer. ${ }^{5}$

For tensor analysis, after seeding for the 1-mm-thick sample we measured the $x$ and $y$ components of the SHG signals by keeping the polarizer, which was placed behind the sample, fixed in the vertical $(x)$ or horizontal $(y)$ position and by rotating the readout-beam polarization. Figure 4 displays the tensor data for the $x$ component. The measured tensor data are fitted according to the polarization expressions in Ref. 14, and the results are also shown in Fig. 4. The best fits gave

$$
\chi_{x x x}^{(2)} / \chi_{x y y}^{(2)} \approx 3, \quad \text { and } \chi_{x x y}^{(2)}=0 .
$$
tained

From the measurements for the y component, we ob-

$$
\chi_{x x x}^{(2)} / \chi_{y y y}^{(2)}>16, \quad \text { and } \chi_{y y x}^{(2)}=0 .
$$

The results of this tensor analysis indicate that for collinearly polarized seed beams the photoinduced $\chi^{(2)}$ in the film exhibited axial symmetry along the seed beam polarization direction.
In the optimum seeding case, we measured the induced second-order susceptibility $\chi_{\text {eff }}$ of the samples by comparing with $\chi_{q}$ of a Y-cut quartz $\left(\chi_{q}=0.8 \mathrm{pm} / \mathrm{V}\right) .{ }^{15}$ The $\chi_{q} / \chi_{q}$ can be written as ${ }^{16}$

$$
\chi_{\mathrm{eff}} / \chi_{q}=\frac{2 L_{q c} T_{q}^{1 / 2} n_{s}^{3 / 2} \cos \theta_{s} \sqrt{I_{s}^{2 \omega} / I_{q}^{2 \omega}}}{\pi L_{s} \operatorname{sinhc}\left[\alpha_{s}^{2 \omega} L_{s} / 2\right] \exp \left(-\alpha_{s}^{2 \omega} L_{s} / 2\right) n_{q}^{3 / 2} T_{s}^{1 / 2}},
$$

where $L_{q c}$ is the coherence length of quartz, $T_{q}$ and $T_{s}$ are the product of the electromagnetic power transmission factors of fundamental and second-harmonic waves for quartz and sample, respectively. Also, $n_{q}$ and $n_{s}$ are the refractive indices of quartz and sample at $1064 \mathrm{~nm}$, respectively; $L_{s}$ and $\theta_{s}$ are, respectively, the sample thickness and the angle between the boundary normal and the direction of phase propagation inside the sample. $I_{s}$ and $I_{q}$ are the SHG signal intensities of the sample and quartz, respectively. The second-order nonlinear optical coefficient $\chi_{\text {eff }}$ was estimated to be $0.38 \mathrm{pm} / \mathrm{V}$. Although this photoinduced $\chi_{\text {eff }}$ is still small because of the lower concentration of azodye doped in the samples, the small absorption coefficient of this sample at $532 \mathrm{~nm}$ should permit the use of bulk samples with greater thickness and higher azodye concentration to increase the SHG conversion efficiency.

We experimentally demonstrated phase-matched SHG in bulk azodye-doped PMMA using the all-optical poling technique. The measurements for the dependence of SHG on the sample thickness showed that the SHG signals increased with the increase in sample thickness, indicating that a $\chi^{(2)}$ grating that satisfied the phase-matching condition for SHG was optically induced in the bulk polymer samples. We expect that this study will promote the application of all-optical poling in photonic devices.

\footnotetext{
${ }^{1}$ Molecular Nonlinear Optics-Material, Physics and Devices, edited by J. Zyss (Academic, San Diego, CA, 1994).

${ }^{2}$ Nonlinear Optical Properties of Polymers, edited by A. J. Heeger, J. Orenstein, and D. R. Ulrich (Materials Research Society, Pittsburgh, PA, 1988).

${ }^{3}$ F. Charra, F. Kajzar, J.-M. Nunzi, P. Raimond, and E. Idiart, Opt. Lett. 18, 941 (1993).

${ }^{4}$ W. Chalupczak, C. Fiorini, F. Charra, J.-M. Nunzi, and P. Raimond, Opt. Commun. 126, 103 (1996).

${ }^{5}$ C. Fiorini, F. Charra, J.-M. Nunzi, and P. Raimond, J. Opt. Soc. Am. B 14, 1984 (1997).

${ }^{6}$ R. Piron, E. Toussaere, D. Josse, S. Brasselet, and J. Zyss, Opt. Lett. 25, 1255 (2000).

${ }^{7}$ A. Apostoluk, D. Chapron, G. Gadret, B. Sahraoui, J.-M. Nunzi, C. Fiorini, and P. Raimond, Opt. Lett. 27, 2028 (2002).

${ }^{8}$ N. Tsutsumi, J. Yamamoto, and W. Sakai, Jpn. J. Appl. Phys. 40, 2264 (2001)

${ }^{9}$ X. Yu, X. Zhong, Q. Li, S. Luo, Y. Chen, Y. Sui, and J. Yin, Opt. Lett. 26, 220 (2001).

${ }^{10}$ C. Fiorini, F. Charra, P. Raimond, A. Lorin, and J.-M. Nunzi, Opt. Lett. 22, 1846 (1997).

${ }^{11}$ J. Si, J. Qiu, K. Kitaoka, and K. Hirao, J. Appl. Phys. 89, 2029 (2001).

${ }^{12}$ Y. Shen and H. Rau, Makromol. Chem. 192, 945 (1991).

${ }^{13}$ J. Si, K. Kitaoka, T. Mitsuyu, P. Ye, and K. Hirao, J. Appl. Phys. 85, 8018 (1999).

${ }^{14}$ T. J. Driscoll and N. M. Lawandy, J. Opt. Soc. Am. B 11, 355 (1994).

${ }^{15}$ K. D. Singer and L. A. King, J. Appl. Phys. 70, 3215 (1991).

${ }^{16}$ M. A. Mortazavi, A. Knoesen, S. T. Kowel, B. G. Higgins, and A. Dienes, J. Opt. Soc. Am. B 6, 733 (1989).
} 